\title{
EchoGéo
}

$10 \mid 2009$

La piraterie

\section{Au voyageur engagé}

Jean Marie Théodat

\section{OpenEdition}

Journals

Édition électronique

URL : https://journals.openedition.org/echogeo/11479

DOI : 10.4000/echogeo. 11479

ISSN : 1963-1197

\section{Éditeur}

Pôle de recherche pour l'organisation et la diffusion de l'information géographique (CNRS UMR 8586)

Référence électronique

Jean Marie Théodat, «Au voyageur engagé », EchoGéo [En ligne], 10 | 2009, mis en ligne le 24 novembre 2009, consulté le 16 septembre 2021. URL : http://journals.openedition.org/echogeo/11479 ; DOI : https://doi.org/10.4000/echogeo.11479

Ce document a été généré automatiquement le 16 septembre 2021

EchoGéo est mis à disposition selon les termes de la licence Creative Commons Attribution - Pas d'Utilisation Commerciale - Pas de Modification 4.0 International (CC BY-NC-ND) 


\title{
Au voyageur engagé
}

\author{
Jean Marie Théodat
}

1 L'apport de Lévi-Strauss aux sciences sociales est tel que sa mort, plutôt que de l'atténuer, n'en ravive que davantage notre sentiment de dette et de gratitude envers son héritage. Il a initié une pensée ajustée à la complexité des problèmes posés à une époque charnière de notre modernité. Son idée qu'au-delà de la différence, qui peut être radicale entre les sociétés, il y a une fondamentale ressemblance des structures qui les organise, reste la clé d'une investigation scientifique sérieuse en matière de sciences humaines et sociales. Cette dialectique du proche et du lointain, du familier et de l'étranger, guide l'enquête de terrain, comme un horizon indépassable et la première limite du chercheur. Cette limite, Lévi-Strauss aujourd'hui l'a franchie. Dans le ciel des idées, il contemple sans doute les structures durables des réalités terrestres avec la satisfaction modeste d'avoir fait son métier, qui fut de voyager et de comprendre.

2 Ce voyageur engagé, la mort le rend à la fois plus proche et plus inaccessible. Il nous laisse en partage une claire conscience des chausse-trappes de notre système de pensée et une grille d'analyse qui éclaire la complexité des cultures du monde. L'hommage à lui rendu dans un précédent cahier ne doit pas nous dédouaner de rappeler encore combien cette disparition mérite que l'on revienne aux sources d'une pensée qui est la clé d'intelligence des structures complexes du terrain.

3 Si la géographie commence souvent par le goût du voyage, il est des géographes fixes. Qui officient par nécessité en lieux clos. Ceux que la situation d'apprendre ou d'enseigner oblige à passer des heures dans des salles pour parler de pays qu'ils ne visiteront probablement jamais. L'idée n'y est pas d'aller sur le terrain, ni d'initier au métier de géographe, mais de transmettre une connaissance vernaculaire dans des salles plus ou moins correctement équipées et dont l'excellence des connexions électroniques ne suffit pas à faire oublier cette condition paradoxale : l'absence de terrain.

4 C'est à la découverte d'une géographie à distance, que nous vous invitons dans la rubrique Sur le Métier, avec un article de François Louveau sur l'enseignement de la géographie dans les collèges, et un article d'Élisabeth Crémieu sur l'enseignement dans les classes préparatoires aux concours d'entrée dans les grandes écoles. Loin du terrain, 
peut-on encore transmettre un savoir à la fois utile et à jour dans les conditions définies par des programmes qui changent et des exercices formels qui exercent moins l'esprit critique qu'ils ne vérifient l'apprentissage de codes successifs? Oui, manifestement! Ce dossier permet d'insister sur la pédagogie spécifique et les évolutions didactiques qui sont le gage de survie de la discipline. Ainsi commence sans doute le goût du lointain : prémices et promesses de voyages.

Christophe Colomb (autre voyageur engagé) lorsqu'il débarqua en 1492 dans l'archipel des Bahamas, crut s'être rendu en Inde, tant ce pays exerçait et continue d'exercer sur notre imagination une fascination où l'exotisme le dispute encore à un désir sincère de comprendre. Depuis, cette erreur toponymique est restée attachée à certains territoires des Amériques où l'indio' désigne parfois indifféremment le Taïno, le Guarani ou le Quetchua. La confusion atteint son comble lorsque certains se croient obligés, pour faire savants, de parler des Indes. Notre propos, c'est d'explorer l'Inde, la vraie.

6 C'est dire que c'est à la découverte d'un nouveau monde que vous invite la rubrique Sur le Terrain : l'Inde, puissance émergente. Car il importe de comprendre les lignes de force d'un pays qui va peser durablement dans les relations internationales. Mettre en lumière les défis auxquels doit faire face l'Inde nouvelle, tel est le propos du dossier coordonné par Aurélie Varrel. Un souci de varier les perspectives dans les articles nous fait aborder ce continent à la fois à l'échelle mondiale de la diaspora indienne, qu'à l'échelle régionale du rayonnement métropolitain de Mumbaï; une égale exigence d'équilibre explique que l'on s'intéresse ici aussi bien aux technologies nouvelles dont ce pays a fait le fer de lance de son émergence économique, qu'au savoir scientifique ancien dont la réhabilitation est en plein essor. Ce cahier rend compte de la revanche historique que semble prendre l'Inde sur son passé de pays dominé, de continent lointain que l'on aborde à l'envers et que l'on croit découvrir dans des pays étranges. L'Inde véritable n'attend pas que l'on vienne la découvrir : elle avance vers nous à pas de géant.

7 L'Inde rayonnera bientôt dans l'océan Indien, il n'est pas sûr cependant que sa puissance suffise aujourd'hui ni demain à contenir un phénomène récurrent : la piraterie dont cet océan est le théâtre, notamment au large de la Somalie. Cette activité met directement en danger les circuits d'approvisionnement de l'Inde obligée d'importer massivement des matières premières passant par les endroits stratégiques où ce fléau existe. La rubrique Sur le Champ donne la parole à des chercheurs et des acteurs dont le souci est de mettre une logique à l'irrationnel, d'éclairer les pénombres d'un monde inverse, où la loi du plus fort est restée la meilleure. La piraterie apparaît comme un défi à la mondialisation qui préconise la fluidité et la sécurité des échanges liée à la liberté de circulation. Ce dossier revient sur le champ où prospèrent les pirates en insistant sur la logique particulière d'une activité violente et lucrative.

La Rubrique Sur L'Ecrit qui clôt ce numéro rend compte des nouveautés de la Bibliothèque de Géographie de la rue Saint-Jacques et renseigne la recherche bibliographique sur le thème du Champ. 